\title{
Domain Decomposition for Discontinuous Galerkin Method with Application to Stokes Flow
}

Piotr Krzyżanowski

Warsaw University, Faculty of Mathematics, Informatics and Mechanics (http://www.mimuw.edu.pl/piotr.krzyzanowski)

Summary. We report on recent results related to domain decomposition methods based on the Discontinuous Galerkin discretizations of Stokes equations. We analyze the efficiency of a block nonoverlapping Schwarz preconditioner based on the approach by Feng and Karakashian [2001]. We also prove the inf-sup stability of a substructuring method.

\section{Introduction}

Discontinuous Galerkin (DG) methods have attained a lot of interest in the past years. These nonconforming finite element methods have several advantages over the classical, conforming elements. For example, the finite elements are very easy to construct and allow the use of nonuniform meshes. Moreover, they still guarantee optimal error estimates, requiring only local regularity of the solution. On the other hand, as compared to the conforming methods, the DG methods introduce more degrees of freedom per grid point.

Recently Filippini and Toselli [2002] proved an inf-sup stability result for a Discontinuous Galerkin approximation of Stokes equations on non-matching grids, while at the same time a series of papers by Toselli [2002], Cockburn et al. [2002], Schötzau et al. [2002] developed the stability and approximation theory of DG methods for the Stokes system.

In this paper, we use a DG discretization of the velocity-pressure formulation of the Stokes equations (1), using macroelements as in Filippini and Toselli [2002]. The variational form of the Stokes equations gives rise to a symmetric operator, and our discretization not necessarily retains this property, depending on the choice of the method.

We consider here two domain decomposition methods for the resulting, possibly nonsymmetric, discrete saddle point problem. First we use the approach of block preconditioning, see Krzyżanowski [2001], Klawonn [1998b], Klawonn [1998a]. Using available results related to nonoverlapping Additive 
Schwarz preconditioning DG discretizations of the second order equations, we prove the convergence rate bounds for the corresponding block preconditioner for the DG Stokes discretization. To the author's knowledge, this is the first such result for domain decomposition preconditioners for DG discretizations of Stokes equations.

Next, we define a substructuring method for the discretization under consideration. We show that the resulting problem has also a saddle point structure. For this problem, we show that the inf-sup constant is independent both of the fine mesh size and of the number of the subdomains. This result is a basis for the analysis of parallel substructuring preconditioners, such as the Neumann-Neumann; this topic, however, is not covered in the present paper.

In the paper, for nonnegative scalars $x, y$, we shall write $x \lesssim y$ if there exits a positive constant $C$, independent of $x, y$ and the mesh parameters $h, H$, such that $x \leq C y$.

\section{DG discretization of the Stokes equation}

Let $\Omega$ be a bounded open polygon in $R^{d}, d=2,3$. The Stokes equations in $\Omega$ read

$$
\begin{aligned}
& -\Delta u+\nabla p=f, \\
& \nabla \cdot u=0
\end{aligned}
$$

where $u=\left(u_{1}, \ldots, u_{d}\right)$ denotes fluid velocity and $p$ is the pressure. For simplicity, we assume homogeneous Dirichlet boundary condition on $u$. The given function $f: \Omega \rightarrow R^{d}$ is the external force.

In what follows, for a domain $D,(\cdot, \cdot)_{D}$ denotes the usual inner product in $L^{2}(D)$ (or, depending on the context, $\left[L^{2}(D)\right]^{d}$ ), while $\langle\cdot, \cdot\rangle_{D}$ denotes the inner product in $L^{2}(\partial D)\left(\left[L^{2}(\partial D)\right]^{d}\right)$. We shall omit the subscript, if the integrals are taken over $\Omega$.

\subsection{Finite element spaces}

Let $\mathcal{T}_{H}$ be a subdivision of $\Omega$ into $N$ disjoint triangles $\Omega_{i}, i=1, \ldots, N$, such that $\bar{\Omega}=\bigcup_{i=1, \ldots, N} \bar{\Omega}_{i}$ and $\mathcal{T}_{H}$ forms an affine, regular triangulation of $\Omega$, with mesh parameter $H$. Further, let $\mathcal{T}_{h}$ denote an affine, shape regular triangulation of $\Omega, \bar{\Omega}=\bigcup_{\kappa \in \mathcal{T}_{h}} \bar{\kappa}$, which is derived from $\mathcal{T}_{H}$ by some refinement procedure. The diameter of a triangle $\kappa \in \mathcal{T}_{h}$ will be denoted by $h_{\kappa}$ and the mesh parameter is $h=\max _{\kappa \in \mathcal{T}_{h}} h_{\kappa}$. By $\mathcal{E}_{h}$ we denote the set of all edges of elements in $\mathcal{T}_{h}$, while $\mathcal{E}_{h}^{0}$ we use to denote the internal edges, that is those not included in $\partial \Omega$; for $e \in \mathcal{E}_{h}$, we also set $\left.h_{e}=\operatorname{diam}(e)\right)$. The set of all edges of elements from $\mathcal{T}_{H}$ will be denoted by $\Gamma$.

In order to formulate our domain decomposition method, we shall use the coarse triangulation $\mathcal{T}_{H}$ of $\Omega$. We shall assume that restricted to each 
subdomain our finite element spaces consist of continuous functions, and that those functions satisfy the inf-sup condition.

Let us begin with the definition of the local finite element spaces,

$$
V_{h}^{r_{i}}\left(\Omega_{i}\right)=\left\{v^{i} \in\left[C\left(\Omega_{i}\right)\right]^{d}: v_{\left.\right|_{\kappa}} \in\left[\operatorname{Pr}_{i}(\kappa)\right]^{d}, \forall \kappa \in \mathcal{T}_{h}, \kappa \subset \Omega_{i}\right\},
$$

and

$$
W_{h}^{q_{i}}\left(\Omega_{i}\right)=\left\{w^{i} \in C\left(\Omega_{i}\right): w_{\left.\right|_{\kappa}} \in P q_{i}(\kappa), \forall \kappa \in \mathcal{T}_{h}, \kappa \subset \Omega_{i}\right\} .
$$

Then, we set global spaces, in which we shall pose our discrete problem,

$$
V_{h}^{H}=\left\{v \in\left[L^{2}(\Omega)\right]^{d}: v_{\left.\right|_{\Omega_{i}}} \in V_{h}^{r_{i}}\left(\Omega_{i}\right), \forall \Omega_{i} \in \mathcal{T}_{H}\right\},
$$

and analogously,

$$
W_{h}^{H}=\left\{w \in L_{0}^{2}(\Omega): w_{\left.\right|_{\Omega_{i}}} \in W_{h}^{q_{i}}\left(\Omega_{i}\right), \forall \Omega_{i} \in \mathcal{T}_{H}\right\} .
$$

For short, we shall denote by $v^{i}$ the restriction of $v \in V_{h}^{H}$ to $\Omega_{i}$. We shall make one more assumption, which relates $V_{h}^{r_{i}}\left(\Omega_{i}\right)$ to $W_{h}^{q_{i}}\left(\Omega_{i}\right)$ :

For $i=1, \ldots, N$, there exist constants $\lambda_{i}$ independent of $h$ such that

$$
\sup _{v \in V_{h}^{r_{i}}\left(\Omega_{i}\right), v \neq 0} \frac{(w, \nabla \cdot v)_{\Omega_{i}}}{|v|_{1, \Omega_{i}}} \gtrsim \lambda_{i}|w|_{0, \Omega_{i}}, \quad \forall w \in W_{h}^{q_{i}}\left(\Omega_{i}\right), \int_{\Omega_{i}} w=0 .
$$

Since the traces of the functions from $V_{h}^{H}$ and $W_{h}^{H}$ (and more generally, from $H^{1}\left(\mathcal{T}_{H}\right)$ ) are double-valued on the interelement interface $\Gamma_{H}^{0}=\Gamma_{H} \backslash \partial \Omega$, where $\Gamma_{H}=\cup_{\Omega_{i} \in \mathcal{T}_{H}} \partial \Omega_{i}$, we shall define, following Arnold et al. [2001/02], their average $\{\cdot\}$ and jump [.] on an edge $e$ shared by two elements $\kappa_{1}, \kappa_{2} \in \mathcal{T}_{h}$, see [Arnold et al., 2001/02, Section 3.1]. The spaces $V_{h}^{H}$ and $W_{h}^{H}$ are equipped with the following norms. For $u \in V_{h}^{H}$ we set [Arnold, 1982, Lemmas 2.2 and $2.1]$

$$
\||| u||^{2}=\sum_{\kappa \in \mathcal{T}_{h}}|u|_{1, \kappa}^{2}+\sum_{e \in \mathcal{E}_{h}} \frac{1}{h_{e}}|[u]|_{0, e}^{2} .
$$

(The corresponding inner product in $V_{h}^{H}$ will be denoted by $((\cdot, \cdot))$.) For $p \in$ $W_{h}^{H}$ we define its norm as the usual $L^{2}$ norm: $|p|^{2}=\sum_{\kappa \in \mathcal{T}_{h}}|p|_{0, \kappa}^{2}$.

\subsection{Discretization}

We use the following discontinuous Galerkin finite element approximation to (1):

Problem 1. Find $\left(u_{h}, p_{h}\right) \in V_{h}^{H} \times W_{h}^{H}$, such that

$$
\begin{aligned}
& A_{h}\left(u_{h}, v\right)+B_{h}\left(v, p_{h}\right)=(f, v), \\
& B_{h}\left(u_{h}, w\right)=0,
\end{aligned}
$$

for all $(v, w) \in V_{h}^{H} \times W_{h}^{H}$. 
Here, we have some freedom in how to choose the form $A_{h}(\cdot, \cdot)$ which approximates the Laplacian, see Schötzau et al. [2002] for a discussion. We allow here for two quite popular choices: the symmetric Interior Penalty method as in Douglas and Dupont [1976] (see also Arnold [1982], Arnold et al. [2001/02]) or the nonsymmetric form, which differs from the previous one by a change in the sign of one boundary term, considered, e.g. in Filippini and Toselli [2002]:

$A_{h}(u, v)=\sum_{\kappa \in \mathcal{T}_{h}}(\nabla u, \nabla v)_{\kappa} \mp \sum_{e \in \mathcal{E}_{h}}\langle[u],\{\nabla v\}\rangle_{e}-\sum_{e \in \mathcal{E}_{h}}\langle\{\nabla u\},[v]\rangle_{e}+\sum_{e \in \mathcal{E}_{h}}\left\langle\mu_{e}[u],[v]\right\rangle_{e}$

The penalty scaling $\mu_{e}$ is a properly chosen function, usually of the form $\mu_{e}=\frac{\delta_{e}}{h_{e}}, e \in \mathcal{E}_{h}$, with constant $\delta_{e}$ large enough to preserve the ellipticity of the original problem. The choice of the sign in the definition above results in different DG methods, as described above, and obviously affects the symmetry of this bilinear form.

The approximate divergence form is defined, see e.g. Toselli [2002],

$$
B_{h}(u, p)=-\sum_{\kappa \in \mathcal{T}_{h}}(p, \nabla \cdot u)_{\kappa}+\sum_{e \in \mathcal{E}_{h}^{0}}\langle\{p\},[u]\rangle_{e}
$$

Let us introduce a stability result for the discrete problem (5):

Lemma 1. The pair $V_{h}^{H} \times W_{h}^{H}$ is inf-sup stable, and the inf-sup constant is independent of both $h$ and $H$.

Proof. A similar theorem has been proved for the rectangular elements, in [Filippini and Toselli, 2002, Theorem 4.1] and this lemma validates it for the case of triangular elements. Under assumptions made throughout the paper, there is a quite straightforward way to prove the above Lemma. The proof of course follows the idea of Boland and Nicolaides [1983]. In view of the local inf-sup assumption, the key point of the proof is to specify a globally stable subspace of $\left(V_{h}^{H}, W_{h}^{H}\right)$. We may use for it e.g. the space $\left(V_{H}^{1}, W_{H}^{0}\right)$, consisting of piecewise linear and piecewise constant functions on $\mathcal{T}_{H}$, respectively, which inf-sup stability can be directly proved (see also Schötzau et al. [2002]). We omit the details due to the lack of space.

\section{Nonoverlapping domain decomposition block preconditioner}

We follow the general idea of Krzyżanowski [2001] (see also Klawonn [1998b] for the symmetric case analysis). Using the natural formulation of the variational discrete problem (5) in the operator form,

$$
\mathcal{M}\left(\begin{array}{l}
u \\
p
\end{array}\right)=\left(\begin{array}{cc}
A & B^{*} \\
B & 0
\end{array}\right)\left(\begin{array}{l}
u \\
p
\end{array}\right)=\left(\begin{array}{l}
F \\
G
\end{array}\right)
$$


we define a block diagonal preconditioner for $\mathcal{M}$

$$
\mathcal{M}_{D}=\left(\begin{array}{cc}
A_{0} & 0 \\
0 & J_{0}
\end{array}\right)
$$

and transform the original system into

Problem 2. Find $(u, p) \in V_{h}^{H} \times W_{h}^{H}$ such that

$$
\mathcal{M}_{D}^{-1} \mathcal{M}^{*} \mathcal{M}_{D}^{-1} \mathcal{M}\left(\begin{array}{l}
u \\
p
\end{array}\right)=\mathcal{M}_{D}^{-1} \mathcal{M}^{*} \mathcal{M}_{D}^{-1}\left(\begin{array}{l}
F \\
G
\end{array}\right)
$$

The operator $\mathcal{P}=\mathcal{M}_{D}^{-1} \mathcal{M}^{*} \mathcal{M}_{D}^{-1} \mathcal{M}$ is positive definite and self-adjoint with respect to the inner product $\left(\mathcal{M}_{D} \cdot, \cdot\right)$ induced by $\mathcal{M}_{D}$, regardless the potential lack of symmetry properties of $\mathcal{M}$, and an iterative method such as the conjugate gradient method can be used to solve this problem efficiently ${ }^{1}$.

The building blocks of the preconditioner $\mathcal{M}_{D}$ will be based on a symmetric nonoverlapping domain decomposition preconditioner for the symmetric DG stiffness matrix. In this way, we will obtain a highly parallelizable, nonoverlapping preconditioner for the whole system. The lack of the overlap is an important feature from the point of view of parallel computer implementation, since this lowers the interprocessor communication cost. Moreover, the symmetry of the preconditioned system will give us a possibility to use cheaper symmetric iterative solvers (on the global level) or direct sparse solvers (on the subdomain level).

For $A_{0}^{-1}$ we choose the nonoverlapping Additive Schwarz preconditioner for a symmetric DG method developed by Feng and Karakashian [2001]. According to [Feng and Karakashian, 2001, Theorem 4.5], we have

$$
\|u \mid\|^{2} \lesssim\left(\left(A_{0} u, u\right)\right) \lesssim \frac{H}{h}\|\| u \|^{2}
$$

We also set, for simplicity, $J_{0}^{-1}=M^{-1}$, where $M$ is the pressure mass matrix operator. Since $M$ is block diagonal, with each block corresponding to a mass matrix assembled on a given substructure, $J_{0}^{-1}$ is perfectly parallelizable across the subdomains and can also be relatively cheaply applied using local sparse solvers. Note also that $J_{0}^{-1}$ could even be further simplified, at the price of reducing its efficiency, e.g. by the mass lumping procedure.

The following theorem estimates the condition number of the preconditioned operator:

Theorem 1. Under the above assumptions,

\footnotetext{
${ }^{1}$ Another approach, if $\mathcal{M}^{*}=\mathcal{M}$, could be to solve $\mathcal{M}_{D}^{-1} \mathcal{M}$ with the conjugate residual method, see Klawonn [1998b]. While the two approaches are comparable in the symmetric case, the symmetrized method can also be applied to DG discretizations which lead to nonsymmetric saddle point problems.
} 


$$
\operatorname{cond}(\mathcal{P}) \lesssim\left(\frac{H}{h}\right)^{2}
$$

regardless of the choice of the sign in $A_{h}(\cdot, \cdot)$ in Section 2.2.

Proof. We use the technique of Krzyżanowski [2001]. Due to stability results for $A_{h}(\cdot, \cdot)$, it is sufficient to check only the influence of the quality of the velocity preconditioner. Let us assume that the preconditioner $A_{0}^{-1}$ satisfies

$$
\left.a_{0}\|\| u\left\|\left.\right|^{2} \leq\left(\left(A_{0} u, u\right)\right) \leq a_{1}\right\|\|u\|\right|^{2},
$$

and $a_{0}<1<a_{1}$. Observe that

$$
\begin{aligned}
\left(\mathcal{M}_{D} \mathcal{P}\left(\begin{array}{l}
u \\
p
\end{array}\right),\left(\begin{array}{c}
u \\
p
\end{array}\right)\right)_{V_{h}^{H} \times W_{h}^{H}} & =\left(\left(A_{0}^{-1}\left(A u+B^{*} p\right), A u+B^{*} p\right)\right)+\left(J_{0}^{-1}(B u-C p), B u-C p\right) \\
& \geq \frac{1}{a_{1}}\left|\left\|A u+B^{*} p\left|\|^{2}+\right| B u-\left.C p\right|^{2} .\right.\right.
\end{aligned}
$$

By the stability, we obtain

$$
\left(\mathcal{M}_{D} \mathcal{P}\left(\begin{array}{l}
u \\
p
\end{array}\right),\left(\begin{array}{l}
u \\
p
\end{array}\right)\right)_{V_{h}^{H} \times W_{h}^{H}} \gtrsim \frac{1}{a_{1}}\left(\left.|||u|\right|^{2}+|p|^{2}\right) \gtrsim \frac{a_{0}}{a_{1}}\left(\mathcal{M}_{D}\left(\begin{array}{l}
u \\
p
\end{array}\right),\left(\begin{array}{c}
u \\
p
\end{array}\right)\right)_{V_{h}^{H} \times W_{h}^{H}} .
$$

Similarly, see Krzyżanowski [2001], we prove the upper bound,

$$
\left(\mathcal{M}_{D} \mathcal{P}\left(\begin{array}{l}
u \\
p
\end{array}\right),\left(\begin{array}{l}
u \\
p
\end{array}\right)\right)_{V_{h}^{H} \times W_{h}^{H}} \lesssim \frac{a_{1}}{a_{0}}\left(\mathcal{M}_{D}\left(\begin{array}{l}
u \\
p
\end{array}\right),\left(\begin{array}{l}
u \\
p
\end{array}\right)\right)_{V_{h}^{H} \times W_{h}^{H}} .
$$

Now the conclusion follows from (9).

\section{Stability of the substructuring method}

In the conforming case, it is known that substructuring preconditioners, such as the FETI or the balancing Neumann-Neumann methods, give rise to only polylogarithmic condition number bound. Thus, in the view of the polynomial in $\frac{H}{h}$ condition bound for the nonoverlapping Additive Schwarz proved in Theorem 1, one can hope for a better behavior of the substructuring methods.

In what follows, we shall give a result, see Pavarino and Widlund [2002], which is a basis for further investigation of the substructuring preconditioners for DG discretizations of Stokes equations: we recognize the substructuring as a specific saddle point problem on the interface and prove its inf-sup stability.

Let us first define the substructuring method, restricting ourselves to the symmetric interior penalty DG discretization. Define $V(\Gamma)$ as the space of restrictions of functions from $V_{h}^{H}$ to the interface $\Gamma_{H}$. We define the local 
(discrete) DG Stokes extension operator $S: V(\Gamma) \rightarrow V_{h}^{H} \times W_{h}^{H}$ as $S u_{\Gamma}=$ $(u, p)$, satisfying $u=u_{\Gamma}$ on the interface $\Gamma$ and on each subdomain $\Omega_{i}$,

$$
\begin{aligned}
& \left(\nabla u_{i}, \nabla v_{i}\right)_{\Omega_{i}}-\left(\operatorname{div} v_{i}, p_{i}\right)_{\Omega_{i}}=\left\langle\left[u_{\Gamma}\right],\left\{\nabla v_{i}\right\}\right\rangle_{\partial \Omega_{i}}, \\
& \left(\operatorname{div} u_{i}, q_{i}\right)_{\Omega_{i}}=\left\langle\left[u_{\Gamma}\right],\left\{q_{i}\right\}\right\rangle_{\partial \Omega_{i}} .
\end{aligned}
$$

Note that, in contrast to the conforming FE discretizations, our Stokes extensions are not homogeneous right hand side problems. Defining $V_{\Gamma}=\{v \in$ $V_{h}^{H}: v=S u_{\Gamma}$, for some $\left.u_{\Gamma} \in V(\Gamma)\right\}$, and $W_{0}=\left\{q \in W_{h}^{H}: q_{\Omega_{i}}=\right.$ const $\left.\forall i\right\}$ we arrive, following the lines of Pavarino and Widlund [2002], at the following form of the Schur complement of the DG Stokes discretization:

Problem 3. Find $\left(u_{\Gamma}, p_{0}\right) \in V_{\Gamma} \times W_{0}$ such that

$$
\begin{aligned}
& A_{h}\left(u_{\Gamma}, v_{\Gamma}\right)+B_{h}\left(v_{\Gamma}, p_{0}\right)=\left(\tilde{F}, v_{\Gamma}\right), \quad \forall v_{\Gamma} \in V_{\Gamma}, \\
& B_{h}\left(u_{\Gamma}, q_{0}\right)=0, \quad \forall q_{0} \in W_{0} .
\end{aligned}
$$

This problem looks similar to the one considered in Pavarino and Widlund [2002] and, despite its different origin, has similar stability property, partly because of good stability properties of the DG discretizations.

Theorem 2. There exists a constant $\beta_{\Gamma}$, independent of $H$ and $h$, such that

$$
\sup _{v_{\Gamma} \in V_{\Gamma}} \frac{B_{h}\left(v_{\Gamma}, q_{0}\right)}{||\left|v_{\Gamma}\right|||} \geq \beta_{\Gamma}\left|q_{0}\right|, \quad \forall q_{0} \in W_{0} .
$$

Proof. Since, see the proof of Lemma 1, the pair $V_{H}^{1} \times W_{H}^{0}$ is inf-sup stable, there exists $\hat{u}$ in $V^{1}\left(\mathcal{T}_{h}\right)$ such that $B_{h}\left(\hat{u}, q_{0}\right)=B_{h}\left(u, q_{0}\right)$ and $\|\hat{u}\||\lesssim\|\| u \||$. Taking $u_{\Gamma}=S\left(\hat{u}_{\left.\right|_{\Gamma}}\right)$ and using the stability of the Stokes extension we see that $v_{\Gamma}=u_{\Gamma}$ satisfies the desired inequality. We skip the details.

\section{Concluding remarks}

Block preconditioners with high level of parallelism and relatively low intersubdomain communication requirements are relatively easy to derive for DG discretizations of the Stokes equations, and their properties directly reflect those of the building blocks for second order elliptic equations. However, existing DD preconditioners for DG Laplacian discretizations feature only $\frac{H}{h}$ condition bound, which makes substructuring preconditioners potentially more attractive. (Another potentially nice feature of using, e.g. the NeumannNeumann alike preconditioners would be, in the case of DG discretizations, that the problem of floating subdomains can be totally avoided.) While the question of their performance remains open, we proved that at least the very Schur complement problem is stable independently of the number of subdomains.

Acknowledgement. This work has partially been supported by the KBN research grant 2 P03A 00524. 


\section{References}

D. N. Arnold. An interior penalty finite element method with discontinuous elements. SIAM J. Numer. Anal., 19(4):742-760, 1982. ISSN 0036-1429.

D. N. Arnold, F. Brezzi, B. Cockburn, and L. D. Marini. Unified analysis of discontinuous Galerkin methods for elliptic problems. SIAM J. Numer. Anal., 39(5):1749-1779 (electronic), 2001/02. ISSN 1095-7170.

J. M. Boland and R. A. Nicolaides. Stability of finite elements under divergence constraints. SIAM J. Numer. Anal., 20(4):722-731, 1983. ISSN 0036-1429.

B. Cockburn, G. Kanschat, D. Schötzau, and C. Schwab. Local discontinuous Galerkin methods for the Stokes system. SIAM J. Numer. Anal., 40(1): 319-343 (electronic), 2002. ISSN 1095-7170.

J. Douglas, Jr. and T. Dupont. Interior penalty procedures for elliptic and parabolic Galerkin methods. In Computing methods in applied sciences (Second Internat. Sympos., Versailles, 1975), pages 207-216. Lecture Notes in Phys., Vol. 58. Springer, Berlin, 1976.

X. Feng and O. A. Karakashian. Two-level additive Schwarz methods for a discontinuous Galerkin approximation of second order elliptic problems. SIAM J. Numer. Anal., 39(4):1343-1365 (electronic), 2001. ISSN 10957170.

L. Filippini and A. Toselli. hp finite element approximations on non-matching grids for the Stokes problem. Technical report, ETH, Zurich, Switzerland, 2002.

A. Klawonn. Block-triangular preconditioners for saddle point problems with a penalty term. SIAM J. Sci. Comput., 19(1):172-184 (electronic), 1998a. ISSN 1095-7197. Special issue on iterative methods (Copper Mountain, CO, 1996).

A. Klawonn. An optimal preconditioner for a class of saddle point problems with a penalty term. SIAM J. Sci. Comput., 19(2):540-552 (electronic), 1998b. ISSN 1095-7197.

P. Krzyżanowski. On block preconditioners for nonsymmetric saddle point problems. SIAM J. Sci. Comp., 23(1):157-169, 2001.

L. F. Pavarino and O. B. Widlund. Balancing Neumann-Neumann methods for incompressible Stokes equations. Comm. Pure Appl. Math., 55(3):302335, 2002. ISSN 0010-3640.

D. Schötzau, C. Schwab, and A. Toselli. Mixed $h p$-DGFEM for incompressible flows. SIAM J. Numer. Anal., 40(6):2171-2194 (electronic) (2003), 2002. ISSN 1095-7170.

A. Toselli. $h p$ discontinuous Galerkin approximations for the Stokes problem. Math. Models Methods Appl. Sci., 12(11):1565-1597, 2002. ISSN 0218-2025. 